\title{
Soliton Solutions and Conservation Laws of a $(3+1)$-Dimensional Nonlinear Evolution Equation
}

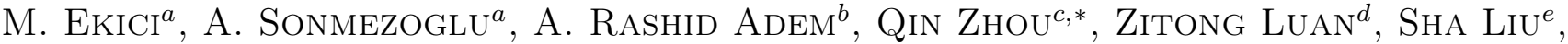 \\ M. MirzazADEH ${ }^{f}$ AND Wenjun LiU $^{g}$ \\ ${ }^{a}$ Department of Mathematics, Faculty of Science and Arts, Yozgat Bozok University, 66100 Yozgat, Turkey \\ ${ }^{b}$ Department of Mathematical Sciences, North-West University, \\ Mafikeng Campus, Private Bag X 2046, Mmabatho 2735, Republic of South Africa \\ ${ }^{c}$ School of Electronics and Information Engineering, \\ Wuhan Donghu University, Wuhan 430212, People's Republic of China \\ ${ }^{d}$ School of Economics and Management, Beijing University of Posts and Telecommunications, \\ Beijing 100876, PR China \\ ${ }^{e}$ Wuhan University Library, Wuhan 430072, People's Republic of China \\ ${ }^{f}$ Department of Engineering Sciences, Faculty of Technology and Engineering, East of Guilan, University of Guilan, \\ PC 44891-63157 Rudsar-Vajargah, Iran \\ ${ }^{g}$ State Key Laboratory of Information Photonics and Optical Communications, and School of Science, \\ P.O. Box 122, Beijing University of Posts and Telecommunications, Beijing 100876, People's Republic of China
}

(Received November 14, 2018; in final form January 17, 2019)

This work studies a new $(3+1)$-dimensional nonlinear model, which was introduced by Abdul-Majid Wazwaz in 2014. This new physical model describes the shallow-water waves and short waves in nonlinear dispersive models. Analytical traveling wave solutions including the solitons and plane wave solutions are derived by using the $G^{\prime} / G$-expansion technique. Moreover, the conserved quantities of this model are also given.

DOI: 10.12693/APhysPolA.135.539

PACS/topics: solitons, conservation laws, extended $G^{\prime} / G$-expansion scheme

\section{Introduction}

Studying the nonlinear evolution equation arising in natural science is very important for us to understand and explain the nonlinear phenomena [1-14]. By the analytical and numerical methods, many researchers constructed travelling wave solutions of some famous nonlinear mathematical physics equations, such as the nonlinear Schrödinger equation in nonlinear optics, the nonlinear Gross-Pitaevskii equation in the Bose-Einstein condensates, the Korteweg-de Vries (KdV) equation in fluid mechanics, and so on. It can be noted that a new $(3+1)$-dimensional nonlinear equation was proposed by Abdul-Majid Wazwaz in 2014, it is an extension version of the $(3+1)$-dimensional KdV equation. This work studies this model by a different method, which is the $G^{\prime} / G$ expansion technique. Some new soliton solutions is reported, and finally the conserved quantities are discussed.

The new $(3+1)$-dimensional nonlinear model that is going to be studied in this paper is given by [1]:

$$
\begin{aligned}
& 3 w_{x z}-\left(2 w_{t}+w_{x x x}-2 w w_{x}\right)_{y}+2\left(w_{x} \partial_{x}^{-1} w_{y}\right)_{x} \\
& \quad+2\left(w \partial_{x}^{-1} w_{y y}\right)_{y}+w_{y z}=0,
\end{aligned}
$$

*corresponding author; e-mail: qinzhou@whu.edu.cn where the inverse operator $\partial_{x}^{-1}$ is defined by:

$$
\left(\partial_{x}^{-1} f\right)(x)=\int_{-\infty}^{x} f(t) \mathrm{d} t,
$$

under the decaying condition at infinity. It should be noted that

$$
\partial_{x} \partial_{x}^{-1}=\partial_{x}^{-1} \partial_{x}=1
$$

We first remove the integral term in (1) by introducing the potential

$$
w(x, y, z, t)=u_{x}(x, y, z, t)
$$

to convert (1) to the equation

$$
\begin{aligned}
& 3 u_{x x z}-\left(2 u_{x t}+u_{x x x x}-2 u_{x} u_{x x}\right)_{y}+2\left(u_{x x} u_{y}\right)_{x} \\
& +2\left(u_{x} u_{y y}\right)_{y}+u_{x y z}=0 .
\end{aligned}
$$

In order to secure soliton solutions to Eq. (5), the following wave variable is employed:

$$
u(x, y, z, t)=U(\eta), \quad \eta=\kappa_{1} x+\kappa_{2} y+\kappa_{3} z-v t,
$$

where $\kappa_{i}(i=1,2,3)$ and $v$ are constants, which are to be determined. Next, inserting (6) into (5), and then integrating the result twice with respect to $\eta$, and choosing constants of integration to zero yields

$$
\begin{aligned}
& \left(2 v \kappa_{1} \kappa_{2}+3 \kappa_{1}^{2} \kappa_{3}+\kappa_{1} \kappa_{2} \kappa_{3}\right) U^{\prime} \\
& \quad+\left(2 \kappa_{1}^{3} \kappa_{2}+\kappa_{1} \kappa_{2}^{3}\right)\left(U^{\prime}\right)^{2}-\kappa_{1}^{4} \kappa_{2} U^{\prime \prime \prime}=0 .
\end{aligned}
$$




\section{Extended $G^{\prime} / G$-expansion scheme}

In this section, extended $G^{\prime} / G$-expansion technique $[2-5]$ is employed to analyze the $(3+1)$-dimensional nonlinear model given by (1). We assume that Eq. (7) has the solution in the form

$$
\begin{aligned}
& U(\eta)=\alpha_{0} \\
& +\sum_{i=1}^{M}\left[\alpha_{i}\left(\frac{G^{\prime}}{G}\right)^{i}+\beta_{i}\left(\frac{G^{\prime}}{G}\right)^{i-1} \sqrt{\sigma\left(1+\frac{1}{\mu}\left(\frac{G^{\prime}}{G}\right)^{2}\right)}\right. \\
& \left.+\gamma_{i}\left(\frac{G^{\prime}}{G}\right)^{-i}+\delta_{i} \frac{\left(\frac{G^{\prime}}{G}\right)^{-i+1}}{\sqrt{\sigma\left(1+\frac{1}{\mu}\left(\frac{G^{\prime}}{G}\right)^{2}\right)}}\right]
\end{aligned}
$$

where $\alpha_{0}, \alpha_{i}, \beta_{i}, \gamma_{i}, \delta_{i}(i=1, \ldots, M)$ are constants to be determined later, $\sigma= \pm 1, M$ is a positive integer, and $G=G(\eta)$ satisfies the following second order linear ODE:

$$
G^{\prime \prime}+\mu G=0,
$$

where $\mu$ is a constant to be determined later. Using the balance method leads to $M=1$. Therefore, the extended $G^{\prime} / G$-expansion approach admits the use of

$$
\begin{gathered}
U(\eta)=\alpha_{0}+\alpha_{1}\left(\frac{G^{\prime}}{G}\right)+\beta_{1} \sqrt{\sigma\left(1+\frac{1}{\mu}\left(\frac{G^{\prime}}{G}\right)^{2}\right)} \\
+\gamma_{1}\left(\frac{G^{\prime}}{G}\right)^{-1}+\delta_{1} \frac{1}{\sqrt{\sigma\left(1+\frac{1}{\mu}\left(\frac{G^{\prime}}{G}\right)^{2}\right)}}
\end{gathered}
$$

Substituting (9) and (10) into the reduced ODE (7), collecting the coefficients of $\left(\frac{G^{\prime}}{G}\right)^{j}$ and $\left(\frac{G^{\prime}}{G}\right)^{j} \sqrt{\sigma\left(1+\frac{1}{\mu}\left(\frac{G^{\prime}}{G}\right)^{2}\right)}$, and solving the resulting system, following sets of solutions are procured where $\mu$ is arbitrary constant:

The first set of parameters is given by

$$
\begin{aligned}
& \beta_{1}=\gamma_{1}=\delta_{1}=0, \quad \alpha_{0}=\alpha_{0}, \quad \alpha_{1}=-\frac{6 \kappa_{1}^{3}}{2 \kappa_{1}^{2}+\kappa_{2}^{2}}, \\
& v=-2 \mu \kappa_{1}^{3}-\frac{\kappa_{3}\left(3 \kappa_{1}+\kappa_{2}\right)}{2 \kappa_{2}} .
\end{aligned}
$$

The second set of parameters is given by

$$
\begin{aligned}
& \alpha_{1}=\beta_{1}=\delta_{1}=0, \quad \alpha_{0}=\alpha_{0}, \quad \gamma_{1}=\frac{6 \mu \kappa_{1}^{3}}{2 \kappa_{1}^{2}+\kappa_{2}^{2}}, \\
& v=-2 \mu \kappa_{1}^{3}-\frac{\kappa_{3}\left(3 \kappa_{1}+\kappa_{2}\right)}{2 \kappa_{2}} .
\end{aligned}
$$

The third set of parameters is given by

$$
\begin{aligned}
& \beta_{1}=\delta_{1}=0, \quad \alpha_{0}=\alpha_{0}, \quad \alpha_{1}=-\frac{6 \kappa_{1}^{3}}{2 \kappa_{1}^{2}+\kappa_{2}^{2}}, \\
& \gamma_{1}=\frac{6 \mu \kappa_{1}^{3}}{2 \kappa_{1}^{2}+\kappa_{2}^{2}}, \quad v=-8 \mu \kappa_{1}^{3}-\frac{\kappa_{3}\left(3 \kappa_{1}+\kappa_{2}\right)}{2 \kappa_{2}} .
\end{aligned}
$$

The fourth set of parameters is given by

$$
\begin{aligned}
& \gamma_{1}=\delta_{1}=0, \quad \alpha_{0}=\alpha_{0}, \quad \alpha_{1}=-\frac{3 \kappa_{1}^{3}}{2 \kappa_{1}^{2}+\kappa_{2}^{2}}, \\
& \beta_{1}= \pm \frac{3 \kappa_{1}^{3} \sqrt{\mu}}{\left(2 \kappa_{1}^{2}+\kappa_{2}^{2}\right) \sqrt{\sigma}}, \\
& v=-\frac{1}{2}\left(\mu \kappa_{1}^{3}+\kappa_{3}+\frac{3 \kappa_{1} \kappa_{3}}{\kappa_{2}}\right) .
\end{aligned}
$$

Using these results, the following exact solutions to Eq. (5) are derived:

When $\mu<0$, the hyperbolic traveling wave solutions are

$$
\begin{aligned}
& u(x, y, z, t)=\alpha_{0}-\frac{6 \kappa_{1}^{3} \sqrt{-\mu}}{2 \kappa_{1}^{2}+\kappa_{2}^{2}} \\
& \quad \times\left[\frac{A_{1} \sinh (\sqrt{-\mu} \eta)+A_{2} \cosh (\sqrt{-\mu} \eta)}{A_{1} \cosh (\sqrt{-\mu} \eta)+A_{2} \sinh (\sqrt{-\mu} \eta)}\right],
\end{aligned}
$$

where $A_{1}, A_{2}$ are arbitrary constants and $\eta=\kappa_{1} x+\kappa_{2} y+$ $\kappa_{3} z+\left(2 \mu \kappa_{1}^{3}+\frac{\kappa_{3}\left(3 \kappa_{1}+\kappa_{2}\right)}{2 \kappa_{2}}\right) t$.

$$
\begin{aligned}
& u(x, y, z, t)=\alpha_{0}-\frac{6 \kappa_{1}^{3} \sqrt{-\mu}}{2 \kappa_{1}^{2}+\kappa_{2}^{2}} \\
& \quad \times\left[\frac{A_{1} \sinh (\sqrt{-\mu} \eta)+A_{2} \cosh (\sqrt{-\mu} \eta)}{A_{1} \cosh (\sqrt{-\mu} \eta)+A_{2} \sinh (\sqrt{-\mu} \eta)}\right]^{-1},
\end{aligned}
$$

where $A_{1}, A_{2}$ are arbitrary constants and $\eta=\kappa_{1} x+\kappa_{2} y+$ $\kappa_{3} z+\left(2 \mu \kappa_{1}^{3}+\frac{\kappa_{3}\left(3 \kappa_{1}+\kappa_{2}\right)}{2 \kappa_{2}}\right) t$.

$$
\begin{aligned}
& u(x, y, z, t)=\alpha_{0}-\frac{6 \kappa_{1}^{3} \sqrt{-\mu}}{2 \kappa_{1}^{2}+\kappa_{2}^{2}} \\
& \quad \times\left\{\frac{A_{1} \sinh (\sqrt{-\mu} \eta)+A_{2} \cosh (\sqrt{-\mu} \eta)}{A_{1} \cosh (\sqrt{-\mu} \eta)+A_{2} \sinh (\sqrt{-\mu} \eta)}\right. \\
& \left.+\left[\frac{A_{1} \sinh (\sqrt{-\mu} \eta)+A_{2} \cosh (\sqrt{-\mu} \eta)}{A_{1} \cosh (\sqrt{-\mu} \eta)+A_{2} \sinh (\sqrt{-\mu} \eta)}\right]^{-1}\right\},
\end{aligned}
$$

where $A_{1}, A_{2}$ are arbitrary constants and $\eta=\kappa_{1} x+\kappa_{2} y+$ $\kappa_{3} z+\left(8 \mu \kappa_{1}^{3}+\frac{\kappa_{3}\left(3 \kappa_{1}+\kappa_{2}\right)}{2 \kappa_{2}}\right) t$.

$$
\begin{aligned}
& u(x, y, z, t)=\alpha_{0}-\frac{3 \kappa_{1}^{3} \sqrt{-\mu}}{2 \kappa_{1}^{2}+\kappa_{2}^{2}} \\
& \quad \times\left\{\frac{A_{1} \sinh (\sqrt{-\mu} \eta)+A_{2} \cosh (\sqrt{-\mu} \eta)}{A_{1} \cosh (\sqrt{-\mu} \eta)+A_{2} \sinh (\sqrt{-\mu} \eta)}\right. \\
& \left.\mp \mathrm{i} \sqrt{1-\left[\frac{A_{1} \sinh (\sqrt{-\mu} \eta)+A_{2} \cosh (\sqrt{-\mu} \eta)}{A_{1} \cosh (\sqrt{-\mu} \eta)+A_{2} \sinh (\sqrt{-\mu} \eta)}\right]^{2}}\right\},
\end{aligned}
$$

where $A_{1}, A_{2}$ are arbitrary constants and $\eta=\kappa_{1} x+\kappa_{2} y+$ $\kappa_{3} z+\frac{1}{2}\left(\mu \kappa_{1}^{3}+\kappa_{3}+\frac{3 \kappa_{1} \kappa_{3}}{\kappa_{2}}\right) t$.

If, however, $\mu>0$, the trigonometric traveling wave solutions are

$$
\begin{aligned}
& u(x, y, z, t)=\alpha_{0}-\frac{6 \kappa_{1}^{3} \sqrt{\mu}}{2 \kappa_{1}^{2}+\kappa_{2}^{2}} \\
& \quad \times\left[\frac{A_{1} \cos (\sqrt{\mu} \eta)-A_{2} \sin (\sqrt{\mu} \eta)}{A_{1} \sin (\sqrt{\mu} \eta)+A_{2} \cos (\sqrt{\mu} \eta)}\right],
\end{aligned}
$$

where $A_{1}, A_{2}$ are arbitrary constants and $\eta=\kappa_{1} x+\kappa_{2} y+$ $\kappa_{3} z+\left(2 \mu \kappa_{1}^{3}+\frac{\kappa_{3}\left(3 \kappa_{1}+\kappa_{2}\right)}{2 \kappa_{2}}\right) t$. 


$$
\begin{aligned}
& u(x, y, z, t)=\alpha_{0}+\frac{6 \kappa_{1}^{3} \sqrt{\mu}}{2 \kappa_{1}^{2}+\kappa_{2}^{2}} \\
& \times\left[\frac{A_{1} \cos (\sqrt{\mu} \eta)-A_{2} \sin (\sqrt{\mu} \eta)}{A_{1} \sin (\sqrt{\mu} \eta)+A_{2} \cos (\sqrt{\mu} \eta)}\right]^{-1},
\end{aligned}
$$

where $A_{1}, A_{2}$ are arbitrary constants and $\eta=\kappa_{1} x+\kappa_{2} y+$ $\kappa_{3} z+\left(2 \mu \kappa_{1}^{3}+\frac{\kappa_{3}\left(3 \kappa_{1}+\kappa_{2}\right)}{2 \kappa_{2}}\right) t$.

$$
\begin{aligned}
& u(x, y, z, t)=\alpha_{0}-\frac{6 \kappa_{1}^{3} \sqrt{\mu}}{2 \kappa_{1}^{2}+\kappa_{2}^{2}} \\
& \quad \times\left\{\frac{A_{1} \cos (\sqrt{\mu} \eta)-A_{2} \sin (\sqrt{\mu} \eta)}{A_{1} \sin (\sqrt{\mu} \eta)+A_{2} \cos (\sqrt{\mu} \eta)}\right. \\
& \left.-\left[\frac{A_{1} \cos (\sqrt{\mu} \eta)-A_{2} \sin (\sqrt{\mu} \eta)}{A_{1} \sin (\sqrt{\mu} \eta)+A_{2} \cos (\sqrt{\mu} \eta)}\right]^{-1}\right\},
\end{aligned}
$$

where $A_{1}, A_{2}$ are arbitrary constants and $\eta=\kappa_{1} x+\kappa_{2} y+$ $\kappa_{3} z+\left(8 \mu \kappa_{1}^{3}+\frac{\kappa_{3}\left(3 \kappa_{1}+\kappa_{2}\right)}{2 \kappa_{2}}\right) t$.

$$
\begin{aligned}
& u(x, y, z, t)=\alpha_{0}-\frac{3 \kappa_{1}^{3} \sqrt{\mu}}{2 \kappa_{1}^{2}+\kappa_{2}^{2}} \\
& \quad \times\left\{\frac{A_{1} \cos (\sqrt{\mu} \eta)-A_{2} \sin (\sqrt{\mu} \eta)}{A_{1} \sin (\sqrt{\mu} \eta)+A_{2} \cos (\sqrt{\mu} \eta)}\right. \\
& \left.\quad \mp \sqrt{1+\left[\frac{A_{1} \cos (\sqrt{\mu} \eta)-A_{2} \sin (\sqrt{\mu} \eta)}{A_{1} \sin (\sqrt{\mu} \eta)+A_{2} \cos (\sqrt{\mu} \eta)}\right]^{2}}\right\},
\end{aligned}
$$

where $A_{1}, A_{2}$ are arbitrary constants and $\eta=\kappa_{1} x+\kappa_{2} y+$ $\kappa_{3} z+\frac{1}{2}\left(\mu \kappa_{1}^{3}+\kappa_{3}+\frac{3 \kappa_{1} \kappa_{3}}{\kappa_{2}}\right) t$.

Finally, if $\mu=0$, the plane wave solutions are

$$
u(x, y, z, t)=\alpha_{0}-\frac{6 \kappa_{1}^{3}}{2 \kappa_{1}^{2}+\kappa_{2}^{2}}\left(\frac{A_{1}}{A_{1} \eta+A_{2}}\right),
$$

where $A_{1}, A_{2}$ are arbitrary constants and $\eta=\kappa_{1} x+\kappa_{2} y+$ $\kappa_{3} z+\left(\frac{\kappa_{3}\left(3 \kappa_{1}+\kappa_{2}\right)}{2 \kappa_{2}}\right) t$.

$$
u(x, y, z, t)=\alpha_{0}-\frac{3 \kappa_{1}^{3}}{2 \kappa_{1}^{2}+\kappa_{2}^{2}}\left(\frac{A_{1}}{A_{1} \eta+A_{2}}\right),
$$

where $A_{1}, \quad A_{2}$ are arbitrary constants and $\eta=\kappa_{1} x+\kappa_{2} y+\kappa_{3} z+\frac{1}{2}\left(\kappa_{3}+\frac{3 \kappa_{1} \kappa_{3}}{\kappa_{2}}\right) t$.

The special cases are as follows:

When $\mu<0$ and $A_{1}^{2}>A_{2}^{2}$, the following bright and singular soliton solutions emerged from (15) to (18), respectively:

$$
\begin{aligned}
& u(x, y, z, t)=\alpha_{0}-\frac{6 \kappa_{1}^{3} \sqrt{-\mu}}{2 \kappa_{1}^{2}+\kappa_{2}^{2}} \\
& \times \tanh \left(\sqrt { - \mu } \left(\kappa_{1} x+\kappa_{2} y+\kappa_{3} z\right.\right. \\
& \left.\left.+\left(2 \mu \kappa_{1}^{3}+\frac{\kappa_{3}\left(3 \kappa_{1}+\kappa_{2}\right)}{2 \kappa_{2}}\right) t\right)+\eta_{0}\right), \\
& u(x, y, z, t)=\alpha_{0}-\frac{6 \kappa_{1}^{3} \sqrt{-\mu}}{2 \kappa_{1}^{2}+\kappa_{2}^{2}} \\
& \quad \times \operatorname{coth}\left(\sqrt { - \mu } \left(\kappa_{1} x+\kappa_{2} y+\kappa_{3} z\right.\right. \\
& \left.\left.+\left(2 \mu \kappa_{1}^{3}+\frac{\kappa_{3}\left(3 \kappa_{1}+\kappa_{2}\right)}{2 \kappa_{2}}\right) t\right)+\eta_{0}\right), \\
& u(x, y, z, t)=\alpha_{0}-\frac{12 \kappa_{1}^{3} \sqrt{-\mu}}{2 \kappa_{1}^{2}+\kappa_{2}^{2}} \\
& \left.\left.\quad+\left(8 \mu \kappa_{1}^{3}+\frac{\kappa_{3}\left(3 \kappa_{1}+\kappa_{2}\right)}{2 \kappa_{2}}\right) t\right)+\eta_{0}\right), \\
& \quad \times \operatorname{coth} 2\left(\sqrt { - \mu } \left(\kappa_{1} x+\kappa_{2} y+\kappa_{3} z\right.\right. \\
& \quad+1
\end{aligned}
$$

where $\eta_{0}=\tanh ^{-1}\left(A_{2} / A_{1}\right)$. Also, setting $A_{1}=0, A_{2} \neq 0$ and $A_{2}=0, A_{1} \neq 0$ in (15)-(18), addition soliton solutions to the model (5) can be secured. However, these are ignored for convenience.

If, however, $\mu>0$, the following periodic wave solutions emerged from (19)-(22), respectively:

$$
\begin{aligned}
& u(x, y, z, t)=\alpha_{0}+\frac{6 \kappa_{1}^{3} \sqrt{\mu}}{2 \kappa_{1}^{2}+\kappa_{2}^{2}} \tan \left(\sqrt{\mu}\left(\kappa_{1} x+\kappa_{2} y+\kappa_{3} z+\left(2 \mu \kappa_{1}^{3}+\frac{\kappa_{3}\left(3 \kappa_{1}+\kappa_{2}\right)}{2 \kappa_{2}}\right) t\right)-\eta_{0}\right), \\
& u(x, y, z, t)=\alpha_{0}-\frac{6 \kappa_{1}^{3} \sqrt{\mu}}{2 \kappa_{1}^{2}+\kappa_{2}^{2}}\left(\sqrt{\mu}\left(\kappa_{1} x+\kappa_{2} y+\kappa_{3} z+\left(2 \mu \kappa_{1}^{3}+\frac{\kappa_{3}\left(3 \kappa_{1}+\kappa_{2}\right)}{2 \kappa_{2}}\right) t\right)-\eta_{0}\right), \\
& u(x, y, z, t)=\alpha_{0}-\frac{12 \kappa_{1}^{3} \sqrt{\mu}}{2 \kappa_{1}^{2}+\kappa_{2}^{2}} 2\left(\sqrt{\mu}\left(\kappa_{1} x+\kappa_{2} y+\kappa_{3} z+\left(8 \mu \kappa_{1}^{3}+\frac{\kappa_{3}\left(3 \kappa_{1}+\kappa_{2}\right)}{2 \kappa_{2}}\right) t\right)-\eta_{0}\right),
\end{aligned}
$$




$$
\begin{aligned}
& u(x, y, z, t)=\alpha_{0}+\frac{3 \kappa_{1}^{3} \sqrt{\mu}}{2 \kappa_{1}^{2}+\kappa_{2}^{2}}\left(\tan \left(\sqrt{\mu}\left(\kappa_{1} x+\kappa_{2} y+\kappa_{3} z+\frac{1}{2}\left(\mu \kappa_{1}^{3}+\kappa_{3}+\frac{3 \kappa_{1} \kappa_{3}}{\kappa_{2}}\right) t\right)-\eta_{0}\right)\right. \\
& \left.\quad \pm \sec \left(\sqrt{\mu}\left(\kappa_{1} x+\kappa_{2} y+\kappa_{3} z+\frac{1}{2}\left(\mu \kappa_{1}^{3}+\kappa_{3}+\frac{3 \kappa_{1} \kappa_{3}}{\kappa_{2}}\right) t\right)-\eta_{0}\right)\right)
\end{aligned}
$$

where $\eta_{0}=\tan ^{-1}\left(A_{1} / A_{2}\right)$. Moreover, setting $A_{1}=0$, $A_{2} \neq 0$ and $A_{2}=0, A_{1} \neq 0$ in (19)-(22), more periodic wave solutions to the model (5) can be acquired. But they are ignored for convenience.

Remark-1: By using (4), soliton and other solutions to the governing Eq. (1) can be obtained.

\section{Conservation laws of (5)}

A conservation law [6, 7] for Eq. (5) is a space-time divergence

$$
D_{t} T^{t}+D_{x} T^{x}+D_{y} T^{y}+D_{z} T^{z}=0,
$$

which holds for all formal solutions $u(t, x, y, z)$ of Eq. (5) where the conserved density $T^{t}$, and the spatial fluxes $T^{x}$, $T^{y}, T^{z}$ are functions of $t, x, y, z, u$, and derivatives of $u$. Furthermore, if there exists a non-trivial differential function $\Lambda$, called a "multiplier" such that $E_{u}(\Lambda G)=0$, then $\Lambda G$ is a total divergence, i.e. $\Lambda G=D_{t} T^{t}+D_{x} T^{x}+D_{y} T^{y}$, for some (conserved) vector $\left[T^{t}, T^{x}, T^{y}, T^{z}\right]$, and $E_{u}$ is the Euler-Lagrange operator. Thus, knowledge of each multiplier $\Lambda$ leads to a conserved vector computed by a homotopy operator. If $u$ and its derivatives tend to zero as $x, y, z$ approaches infinity, the conserved quantities are obtained by $\int_{-\infty}^{\infty} \int_{-\infty}^{\infty} \int_{-\infty}^{\infty} T^{t} \mathrm{~d} x \mathrm{~d} y \mathrm{~d} z$.

For (5), we obtain a multiplier $\Lambda$, that is given by

$$
\begin{aligned}
\Lambda & =f_{1}(t) u+x f_{4}(t, z)+y f_{3}(t, z)+f_{2}(t, z) \\
& +\frac{1}{2} f_{5}(t)\left(y^{2}-x^{2}\right)+\frac{1}{4} x^{2} f_{1}^{\prime},
\end{aligned}
$$

where $f_{i}, i=1,2,3,4,5$ are arbitrary functions. Thus, corresponding to the above multiplier we have the following conservation laws of (5):

$$
D_{t} T_{1}^{t}+D_{x} T_{1}^{x}+D_{y} T_{1}^{y}+D_{z} T_{1}^{z}=0,
$$

where

$$
\begin{aligned}
& T_{1}^{t}=\frac{1}{6}\left(2 f_{1}(t) w \int w_{y} \mathrm{~d} x-4 f_{1}(t) w_{y} \int w \mathrm{~d} x\right. \\
& \left.\quad+x^{2}\left(-f_{1}^{\prime}\right) w_{y}+x f_{1}^{\prime} \int w_{y} \mathrm{~d} x\right),
\end{aligned}
$$

$$
\begin{aligned}
& T_{1}^{x}=\frac{1}{360}\left[30 f_{1}^{\prime} x^{2} \int w_{y z} \mathrm{~d} x+45 f_{1}^{\prime} x^{2}\left(\int w_{y} \mathrm{~d} x\right)\left(\int w_{y y} \mathrm{~d} x\right)+45 f_{1}^{\prime} x^{2}\left(\int w \mathrm{~d} x\right)\left(\int w_{y y y} \mathrm{~d} x\right)\right. \\
& \quad-60 f_{1}^{\prime} x^{2} \int w_{t y} \mathrm{~d} x+30 f_{1}^{\prime \prime} x^{2} \int w_{y} \mathrm{~d} x+180 f_{1}^{\prime} w_{z} x^{2}+120 w f_{1}^{\prime} w_{y} x^{2}+150 f_{1}^{\prime} w_{x} x^{2} \int w_{y} \mathrm{~d} x \\
& \quad-30 f_{1}^{\prime} w_{x y} x^{2} \int w \mathrm{~d} x-72 f_{1}^{\prime} w_{x x y} x^{2}-180 f_{1}^{\prime} x \int w_{z} \mathrm{~d} x-240 w f_{1}^{\prime} x \int w_{y} \mathrm{~d} x+120 f_{1}^{\prime} w_{y} x \int w \mathrm{~d} x \\
& +108 f_{1}^{\prime} w_{x y} x-400 w^{2} f_{1}(t) \int w_{y} \mathrm{~d} x-60 f_{1}(t)\left(\int w_{z} \mathrm{~d} x\right)\left(\int w_{y} \mathrm{~d} x\right) \\
& +120 f_{1}(t)\left(\int w \mathrm{~d} x\right)\left(\int w_{y z} \mathrm{~d} x\right)+120 f_{1}(t)\left(\int w \mathrm{~d} x\right)^{2}\left(\int w_{y y y} \mathrm{~d} x\right) \\
& +120 f_{1}(t)\left(\int w_{y} \mathrm{~d} x\right)\left(\int w_{t} \mathrm{~d} x\right)-240 f_{1}(t)\left(\int w \mathrm{~d} x\right)^{2}\left(\int w_{t y} \mathrm{~d} x\right)-360 w f_{1}(t) \int w_{z} \mathrm{~d} x \\
& +120 f_{1}^{\prime}\left(\int w \mathrm{~d} x\right)\left(\int w_{y} \mathrm{~d} x\right)+720 f_{1}(t) w_{z} \int w \mathrm{~d} x+640 w f_{1}(t) w_{y} \int w \mathrm{~d} x-72 f_{1}^{\prime} w_{y} \\
& +560 f_{1}(t) w_{x}\left(\int w \mathrm{~d} x\right)\left(\int w_{y} \mathrm{~d} x\right)-144 f_{1}(t) w_{y} w_{x}-80 f_{1}(t) w_{x y}\left(\int w \mathrm{~d} x\right)^{2}+216 w f_{1}(t) w_{x y} \\
& \left.+72 f_{1}(t) w_{x x} \int w_{y} \mathrm{~d} x-288 f_{1}(t) w_{x x y} \int w \mathrm{~d} x\right],
\end{aligned}
$$




$$
\begin{aligned}
& T_{1}^{y}=\frac{1}{360}\left[90 x^{2} f_{1}^{\prime} w_{x} w+135 x^{2} f_{1}^{\prime} w \int w_{y y} \mathrm{~d} x-45 x^{2} f_{1}^{\prime} w_{y y} \int w \mathrm{~d} x+30 x^{2} f_{1}^{\prime} w_{x x} \int w \mathrm{~d} x\right. \\
& -90 x f_{1}^{\prime}\left(\int w_{y y} \mathrm{~d} x\right)\left(\int w \mathrm{~d} x\right)-60 x f_{1}^{\prime} w_{x} \int w \mathrm{~d} x+72 f_{1}(t) w_{x x} w-240 f_{1}(t) w\left(\int w_{y} \mathrm{~d} x\right)^{2} \\
& -60 f_{1}(t) w \int w_{z} \mathrm{~d} x+480 f_{1}(t) w\left(\int w_{y y} \mathrm{~d} x\right)\left(\int w \mathrm{~d} x\right)+120 f_{1}(t) w \int w_{t} \mathrm{~d} x+240 f_{1}(t) w_{x} w \int w \mathrm{~d} x \\
& +120 f_{1}(t) w_{z} \int w \mathrm{~d} x+240 f_{1}(t) w_{y}\left(\int w_{y} \mathrm{~d} x\right)\left(\int w \mathrm{~d} x\right)-120 f_{1}(t) w_{y y}\left(\int w \mathrm{~d} x\right)^{2}+80 f_{1}(t) w_{x x}\left(\int w \mathrm{~d} x\right)^{2} \\
& -72 f_{1}(t) w_{x x x} \int w \mathrm{~d} x-240 f_{1}(t) w_{t} \int w \mathrm{~d} x+30 x^{2} f_{1}^{\prime \prime} w-60 x f_{1}^{\prime} w^{2}-120 x f_{1}^{\prime \prime} \int w \mathrm{~d} x-80 f_{1}(t) w^{3}-60 x^{2} f_{1}^{\prime} w_{t} \\
& \left.+60 x f_{1}^{\prime} \int w_{t} \mathrm{~d} x-36 f_{1}(t) w_{x}^{2}+30 x^{2} f_{1}^{\prime} w_{z}-18 x^{2} f_{1}^{\prime} w_{x x x}-30 x f_{1}^{\prime} \int w_{z} \mathrm{~d} x-36 f_{1}^{\prime} w_{x}+36 x f_{1}^{\prime} w_{x x}\right], \\
& T_{1}^{z}=\frac{1}{12}\left(-2 f_{1}(t) w \int w_{y} \mathrm{~d} x+4 f_{1}(t) w_{y} \int w \mathrm{~d} x+12 f_{1}(t) w_{x} \int w \mathrm{~d} x-6 x f_{1}^{\prime} w+6 f_{1}^{\prime} \int w \mathrm{~d} x\right. \\
& \left.-6 f_{1}(t) w^{2}+x^{2} f_{1}^{\prime} w_{y}+3 x^{2} f_{1}^{\prime} w_{x}-x f_{1}^{\prime} \int w_{y} \mathrm{~d} x\right) \\
& D_{t} T_{2}^{t}+D_{x} T_{2}^{x}+D_{y} T_{2}^{y}+D_{z} T_{2}^{z}=0, \quad \text { where } \quad T_{2}^{t}=-\frac{2}{3} w_{y} f_{2}(t, z), \\
& T_{2}^{x}=\frac{1}{30}\left[40 w_{y} f_{2}(t, z) w-24 f_{2}(t, z) w_{x x y}-30 f_{2 z} w+10 f_{2}(t, z) \int w_{y z} \mathrm{~d} x+15 f_{2}(t, z)\left(\int w_{y} \mathrm{~d} x\right)\left(\int w_{y y} \mathrm{~d} x\right)\right. \\
& +15 f_{2}(t, z)\left(\int w_{y y y} \mathrm{~d} x\right)\left(\int w \mathrm{~d} x\right)-20 f_{2}(t, z) \int w_{t y} \mathrm{~d} x+50 w_{x} f_{2}(t, z) \int w_{y} \mathrm{~d} x-10 f_{2}(t, z) w_{x y} \int w \mathrm{~d} x \\
& \left.+60 w_{z} f_{2}(t, z)+10 f_{2 t} \int w_{y} \mathrm{~d} x-5 f_{2 z} \int w_{y} \mathrm{~d} x\right] \\
& T_{2}^{y}=\frac{1}{30}\left(30 w_{x} f_{2}(t, z) w-5 f_{2 z} w+10 f_{2 t} w+45 f_{2}(t, z) w \int w_{y y} \mathrm{~d} x-15 w_{y y} f_{2}(t, z) \int w \mathrm{~d} x\right. \\
& \left.+10 w_{x x} f_{2}(t, z) \int w \mathrm{~d} x-6 w_{x x x} f_{2}(t, z)+10 w_{z} f_{2}(t, z)-20 w_{t} f_{2}(t, z)\right), \\
& T_{2}^{z}=\frac{1}{3}\left(3 w_{x} f_{2}(t, z)+w_{y} f_{2}(t, z)\right) \\
& D_{t} T_{3}^{t}+D_{x} T_{3}^{x}+D_{y} T_{3}^{y}+D_{z} T_{3}^{z}=0, \quad \text { where } T_{3}^{t}=\frac{1}{3}\left(f_{3}(t, z) w-2 y w_{y} f_{3}(t, z)\right), \\
& T_{3}^{x}=\frac{1}{30}\left[-30 y f_{3 z} w+40 y w_{y} f_{3}(t, z) w-24 y f_{3}(t, z) w_{x x y}+10 y f_{3}(t, z) \int w_{y z} \mathrm{~d} x\right. \\
& -15 f_{3}(t, z)\left(\int w_{y y} \mathrm{~d} x\right)\left(\int w \mathrm{~d} x\right)+15 y f_{3}(t, z)\left(\int w_{y} \mathrm{~d} x\right)\left(\int w_{y y} \mathrm{~d} x\right)+15 y f_{3}(t, z)\left(\int w_{y y y} \mathrm{~d} x\right)\left(\int w \mathrm{~d} x\right) \\
& -20 y f_{3}(t, z) \int w_{t y} \mathrm{~d} x+10 f_{3 z} \int w \mathrm{~d} x-20 f_{3 t} \int w \mathrm{~d} x-10 w_{x} f_{3}(t, z) \int w \mathrm{~d} x+50 y w_{x} f_{3}(t, z) \int w_{y} \mathrm{~d} x \\
& -10 y f_{3}(t, z) w_{x y} \int w \mathrm{~d} x+6 w_{x x} f_{3}(t, z)-5 f_{3}(t, z) \int w_{z} \mathrm{~d} x+10 f_{3}(t, z) \int w_{t} \mathrm{~d} x+60 y w_{z} f_{3}(t, z)-10 f_{3}(t, z) w^{2} \\
& \left.+10 y f_{3 t} \int w_{y} \mathrm{~d} x-5 y f_{3 z} \int w_{y} \mathrm{~d} x\right]
\end{aligned}
$$




$$
\begin{aligned}
& T_{3}^{y}=\frac{1}{30}\left(30 y w_{x} f_{3}(t, z) w-6 y w_{x x x} f_{3}(t, z)-5 y f_{3 z} w+10 y f_{3 t} w-30 f_{3}(t, z) w \int w_{y} \mathrm{~d} x+45 y f_{3}(t, z) w \int w_{y y} \mathrm{~d} x\right. \\
& \left.\quad+30 w_{y} f_{3}(t, z) \int w \mathrm{~d} x-15 y w_{y y} f_{3}(t, z) \int w \mathrm{~d} x+10 y w_{x x} f_{3}(t, z) \int w \mathrm{~d} x+10 y w_{z} f_{3}(t, z)-20 y w_{t} f_{3}(t, z)\right) \\
& T_{3}^{z}=\frac{1}{6}\left(6 y w_{x} f_{3}(t, z)+2 y w_{y} f_{3}(t, z)+f_{3}(t, z)(-w)\right), \\
& D_{t} T_{4}^{t}+D_{x} T_{4}^{x}+D_{y} T_{4}^{y}+D_{z} T_{4}^{z}=0, \\
& \quad \text { where } T_{4}^{t}=\frac{1}{3}\left(f_{4}(t, z) \int w_{y} \mathrm{~d} x-2 x w_{y} f_{4}(t, z)\right), \\
& \quad+15 x f_{4}(t, z)\left(\int w_{y} \mathrm{~d} x\right)\left(\int w_{y y} \mathrm{~d} x\right)+15 x f_{4}(t, z)\left(\int w_{y y y} \mathrm{~d} x\right)\left(\int w \mathrm{~d} x\right)-20 x f_{4}(t, z) \int w_{t y} \mathrm{~d} x \\
& \quad-40 f_{4}(t, z) w \int w_{y} \mathrm{~d} x+20 w_{y} f_{4}(t, z) \int w \mathrm{~d} x+50 x w_{x} f_{4}(t, z) \int w_{y} \mathrm{~d} x-10 x f_{4}(t, z) w_{x y} \int w \mathrm{~d} x \\
& \left.\quad+60 f_{4 z} \int w \mathrm{~d} x+60 x w_{z} f_{4}(t, z)-30 f_{4}(t, z) \int w_{z} \mathrm{~d} x+10 x f_{4 t} \int w_{y} \mathrm{~d} x-5 x f_{4 z} \int w_{y} \mathrm{~d} x\right] \\
& \left.\quad+10 f_{4}(t, z) w^{2}\right], \\
& \quad+10 x w_{z} f_{4}(t, z)+6 w_{x x} f_{4}(t, z)-6 x w_{x x} f_{4}(t, z)-20 x w_{t} f_{4}(t, z)-5 f_{4}(t, z) \int w_{z} \mathrm{~d} x+10 f_{4}(t, z) \int w_{t} \mathrm{~d} x \\
& T_{4}^{z}=\frac{1}{6}\left(2 x w_{y} f_{4}(t, z)-f_{4}(t, z) \int w_{y} \mathrm{~d} x+6 x w_{x} f_{4}(t, z)-6 f_{4}(t, z) w\right) \\
& \quad+10 f_{4 z} \int w \mathrm{~d} x-20 f_{4 t} \int w \mathrm{~d} x-15 x w_{y y} f_{4}(t, z) \int w \mathrm{~d} x-10 w_{x} f_{4}(t, z) \int w \mathrm{~d} x+10 x w_{x x} f_{4}(t, z) \int w \mathrm{~d} x \\
& D_{t} T_{5}^{t}+D_{x} T_{5}^{x}+D_{y} T_{5}^{y}+D_{z} T_{5}^{z}=0, \\
& \quad-5 x f_{4 z} w+10 x f_{4 t} w+30 x w_{x} f_{4}(t, z) w+45 x f_{4}(t, z) w \int w_{y y} \mathrm{~d} x-15 f_{4}(t, z)\left(\int w_{y y} \mathrm{~d} x\right)\left(\int w \mathrm{~d} x\right)
\end{aligned}
$$$$
\text { where } T_{5}^{t}=\frac{1}{3}\left(y f_{5}(t) w+x^{2} f_{5}(t) w_{y}-x f_{5}(t) \int w_{y} \mathrm{~d} x-y^{2} f_{5}(t) w_{y}\right) \text {, }
$$$$
T_{5}^{x}=\frac{1}{60}\left[-40 x^{2} f_{5}(t) w_{y} w-15 x^{2} f_{5}(t)\left(\int w_{y y y} \mathrm{~d} x\right)\left(\int w \mathrm{~d} x\right)+10 x^{2} f_{5}(t) w_{x y} \int w \mathrm{~d} x+40 y^{2} f_{5}(t) w_{y} w\right.
$$$$
+15 y^{2} f_{5}(t)\left(\int w_{y y y} \mathrm{~d} x\right)\left(\int w \mathrm{~d} x\right)-10 y^{2} f_{5}(t) w_{x y} \int w \mathrm{~d} x+80 x f_{5}(t) w \int w_{y} \mathrm{~d} x-40 x f_{5}(t) w_{y} \int w \mathrm{~d} x
$$$$
-60 f_{5}(t)\left(\int w_{y} \mathrm{~d} x\right)\left(\int w \mathrm{~d} x\right)-30 y f_{5}(t)\left(\int w_{y y} \mathrm{~d} x\right)\left(\int w \mathrm{~d} x\right)-20 y f_{5}(t) w_{x} \int w \mathrm{~d} x-40 y f_{5}^{\prime} \int w \mathrm{~d} x
$$$$
-20 y f_{5}(t) w^{2}-10 x^{2} f_{5}(t) \int w_{y z} \mathrm{~d} x+24 x^{2} f_{5}(t) w_{x x y}-15 x^{2} f_{5}(t)\left(\int w_{y} \mathrm{~d} x\right)\left(\int w_{y y} \mathrm{~d} x\right)+20 x^{2} f_{5}(t) \int w_{t y} \mathrm{~d} x
$$ 


$$
\begin{aligned}
& -50 x^{2} f_{5}(t) w_{x} \int w_{y} \mathrm{~d} x-60 x^{2} f_{5}(t) w_{z}+10 y^{2} f_{5}(t) \int w_{y z} \mathrm{~d} x-24 y^{2} f_{5}(t) w_{x x y} \\
& +15 y^{2} f_{5}(t)\left(\int w_{y} \mathrm{~d} x\right)\left(\int w_{y y} \mathrm{~d} x\right)-20 y^{2} f_{5}(t) \int w_{t y} \mathrm{~d} x+50 y^{2} f_{5}(t) w_{x} \int w_{y} \mathrm{~d} x-10 y f_{5}(t) \int w_{z} \mathrm{~d} x \\
& -36 x f_{5}(t) w_{x y}+12 y f_{5}(t) w_{x x}+20 y f_{5}(t) \int w_{t} \mathrm{~d} x+60 x f_{5}(t) \int w_{z} \mathrm{~d} x+60 y^{2} f_{5}(t) w_{z}+24 f_{5}(t) w_{y} \\
& \left.-10 x^{2} f_{5}^{\prime} \int w_{y} \mathrm{~d} x+10 y^{2} f_{5}^{\prime} \int w_{y} \mathrm{~d} x\right] \\
& T_{5}^{y}=\frac{1}{60}\left[-30 x^{2} f_{5}(t) w_{x} w-45 x^{2} f_{5}(t) w \int w_{y y} \mathrm{~d} x+15 x^{2} f_{5}(t) w_{y y} \int w \mathrm{~d} x-10 x^{2} f_{5}(t) w_{x x} \int w \mathrm{~d} x+30 y^{2} f_{5}(t) w_{x} w\right. \\
& +45 y^{2} f_{5}(t) w \int w_{y y} \mathrm{~d} x-15 y^{2} f_{5}(t) w_{y y} \int w \mathrm{~d} x+10 y^{2} f_{5}(t) w_{x x} \int w \mathrm{~d} x+30 x f_{5}(t)\left(\int w_{y y} \mathrm{~d} x\right)\left(\int w \mathrm{~d} x\right) \\
& +20 x f_{5}(t) w_{x} \int w \mathrm{~d} x-60 y f_{5}(t) w \int w_{y} \mathrm{~d} x+60 y f_{5}(t) w_{y} \int w \mathrm{~d} x-10 x^{2} f_{5}^{\prime} w+10 y^{2} f_{5}^{\prime} w+40 x f_{5}^{\prime} \int w \mathrm{~d} x \\
& +20 x f_{5}(t) w^{2}+40 f_{5}(t) w \int w \mathrm{~d} x-10 x^{2} f_{5}(t) w_{z}+6 x^{2} f_{5}(t) w_{x x x}+20 x^{2} f_{5}(t) w_{t}-6 y^{2} f_{5}(t) w_{x x x} \\
& \left.+10 x f_{5}(t) \int w_{z} \mathrm{~d} x-12 x f_{5}(t) w_{x x}+12 f_{5}(t) w_{x}-20 x f_{5}(t) \int w_{t} \mathrm{~d} x+10 y^{2} f_{5}(t) w_{z}-20 y^{2} f_{5}(t) w_{t}\right], \\
& T_{5}^{z}=\frac{1}{6}\left(6 x f_{5}(t) w-y f_{5}(t) w-6 f_{5}(t) \int w \mathrm{~d} x-x^{2} f_{5}(t) w_{y}-3 x^{2} f_{5}(t) w_{x}+3 y^{2} f_{5}(t) w_{x}\right. \\
& \left.+x f_{5}(t) \int w_{y} \mathrm{~d} x+y^{2} f_{5}(t) w_{y}\right) .
\end{aligned}
$$

Remark-2: Due to the presence of the arbitrary functions, in the multiplier, one can obtain an infinitely many conservation laws of (5).

\section{Conclusions}

The new $(3+1)$-dimensional nonlinear model has been investigated analytically. Based on the $G^{\prime} / G$ expansion method, the hyperbolic travelling wave solutions, trigonometric travelling wave solutions, plane wave solutions, bright and singular soliton solutions, and periodic wave solutions are presented. Finally, the conservation laws are discussed.

\section{Acknowledgments}

The work by Qin Zhou was supported by the National Natural Science Foundation of China (Grant Nos. 11705130 and 11547149). This author was also sponsored by the Chutian Scholar Program of Hubei Government in China.

\section{References}

[1] A.M. Wazwaz, Centr. Europ. J. Eng. 4, 352 (2014).

[2] S. Guo, Y. Zhou, Appl. Math. Computat. 215, 3214 (2010).

[3] M. Hayek, Appl. Math. Computat. 217, 212 (2010).
[4] Q. Zhou, M. Ekici, A. Sonmezoglu, M. Mirzazadeh, Optik 127, 6277 (2016).

[5] A. Sonmezoglu, M. Ekici, M. Moradi, M. Mirzazadeh, Q. Zhou, Optik 128, 77 (2017).

[6] L.D. Moleleki, B. Muatjetjeja, A.R. Adem, Nonlin. Dyn. 87, 2187 (2017).

[7] A.R. Adem, Int. J. Mod. Phys. B 30, 1640001 (2016).

[8] K. Ali, S.T.R. Rizvi, A. Khalil, M. Younis, Optik 172, 657 (2018).

[9] S.T.R. Rizvi, K. Ali, U. Akram, M. Younis, Optik 168, 27 (2018).

[10] S. Ali, M. Younis, M.O. Ahmad, S.T.R. Rizvi, Opt. Quant. Electron. 50, 266 (2018).

[11] X. Liu, H. Triki, Q. Zhou, W. Liu, A. Biswas, Nonlin. Dyn. 94, 703 (2018).

[12] X. Liu, H. Triki, Q. Zhou, M.D. Mirzazadeh, W. Liu, A. Biswas, M. Belic, Nonlin. Dyn. 95, 143 (2019).

[13] W. Liu, Y. Zhang, H. Triki, M. Mirzazadeh, M. Ekici, Q. Zhou, A. Biswas, M. Belic, Nonlin. Dyn. 95, 557 (2019).

[14] C. Yang, W. Liu, Q. Zhou, D. Mihalache, B.A. Malomed, Nonlin. Dyn. 95, 369 (2019). 